\title{
¿ scontem \\ Differential Protein Expression of Osteoclastogenic Factors in Odontogenic Cysts and Tumors
}

\section{Vildeman Almeida Junior}

Federal University of Bahia: Universidade Federal da Bahia https://orcid.org/0000-0002-9179-6467

\section{Éder Gerardo Santos Leite}

Federal University of Bahia: Universidade Federal da Bahia

\section{Marcus Almeida}

Federal University of Bahia: Universidade Federal da Bahia

\section{Jurema Freire Lisboa de Castro}

Faculty of Dentistry of Recife: Faculdade de Odontologia do Recife

\section{Roseana Almeida Freitas}

Federal University of Rio Grande do Norte: Universidade Federal do Rio Grande do Norte

Flávia Caló Aquino Xavier

Federal University of Bahia: Universidade Federal da Bahia

\section{Andreia Leal Figueiredo}

Federal University of Bahia: Universidade Federal da Bahia

Jean Nunes Santos

Federal University of Bahia: Universidade Federal da Bahia

Águida Cristina Gomes Henriques ( $\sim$ aguidacgh@gmail.com )

Federal University of Bahia: Universidade Federal da Bahia

\section{Research}

Keywords: bone resorption, bone cysts, ameloblastoma, biologic markers, immunohistochemistry, osteoclast

Posted Date: October 23rd, 2020

DOl: https://doi.org/10.21203/rs.3.rs-95728/v1

License: (c) (i) This work is licensed under a Creative Commons Attribution 4.0 International License. Read Full License 


\section{Abstract}

Background: The osteolytic activity of odontogenic cysts and tumors is directly associated with their growth and aggressiveness. Proteins expressed by epithelial and mesenchymal cells can influence this biological event differently in indolent cystic lesions, aggressive cystic lesions, and odontogenic tumors.

Methods: The objective of this study was to compare the immunohistochemical expression of factors that stimulate RANKL (Receptor activator of nuclear factor kappa-B ligand), CatK (cathepsin K) and MMP8 (Matrix Metallopeptidase 8) and inhibit OPG (osteoprotegerin) osteoclastogenesis between dentigerous cyst (DC), glandular odontogenic cyst (GOC), odontogenic keratocyst (OKC), and ameloblastoma (AB). Paraffin-embedded sections of 9 DCs, 9 GOCs, 20 OKCs, 21 ABs, and 4 dental follicles (DFs) were submitted to immunohistochemistry. The immunoreactivity in epithelium and connective tissue was analyzed semi-quantitatively and quantitatively, respectively. Immunoexpression of the proteins was observed in epithelial and mesenchymal cells of all lesions studied.

Results: The expression of RANKL and CatK was higher in OKC, AB, and GOC $(p<0.005)$. Higher expression of OPG was found in DF and DC compared to the other markers $(p<0.005)$. MMP8 expression was high in GOC e OKC.

Conclusions: This study demonstrated the differential expression of factors that inhibit and stimulate bone resorption during the development of DC, GOC, OKC and AB. Higher expression of RANKL and CatK was observed in more aggressive lesions. OPG appears to be one of the molecules responsible for the slower growth of DC.

\section{Background}

The development, progression and variable biological behavior of odontogenic lesions are related to their osteolytic activity [1]. The altered expression of proteins that stimulate or inhibit osteoclastogenesis, associated with the activity of extracellular matrix metalloproteinases (MMPs), may influence the behavior of these lesions $[2,3,4]$. Bone resorption is the main biological event responsible for the progression and aggressiveness of odontogenic lesions, which depends on the formation and activation of osteoclasts. According Chen et al. (2014), Guerrini and Takayanagi (2014) and Sun et al. (2015) several protein factors have been shown to participate in the regulation of bone metabolism [5-7]. These factors activate or inhibit osteoblasts and osteoclasts.

The identification of the triad consisting of receptor activator of nuclear factor kappa- $\beta$ (RANK), its ligand (RANKL), and osteoprotegerin (OPG) has made a significant contribution to the understanding of the biology of bone remodeling. In addition, other molecules participate in the degradation of the organic and inorganic matrix of bone tissue, favoring osteoclastogenesis $[8,9]$. RANK and RANKL have been associated with higher osteolytic activity and are considered factors that stimulate bone resorption [3]. On the other hand, OPG exerts the opposite effect and is known as an inhibitor of osteoclastogenesis. The RANK/RANKL/OPG ratio has been associated with the development of odontogenic lesions [10]. 
Cathepsin K (CatK) and MMP8 are important proteases that participate in the degradation of bone matrix protein components, especially collagen I. The role of CatK and MMP8 in the development of odontogenic lesions has been little studied and the activity of these enzymes associated with the RANK/RANKL/OPG triad can potentiate bone resorption and contribute to the growth of odontogenic lesions $[2,3,10,11]$.

\section{Material And Methods}

Since factors that stimulate and inhibit osteoclastogenesis can determine differences in growth between cysts and odontogenic tumors, the present study compared the protein expression of RANKL, CatK, MMP8 and OPG between indolent odontogenic cystic lesions (dentigerous cyst - DC), aggressive odontogenic cystic lesions (glandular odontogenic cyst - GOC, odontogenic keratocyst - OKC) and odontogenic tumors (ameloblastoma - AB).

The sample consisted of 63 paraffin-embedded tissue specimens, including 9 DCs, 9 GOCs (7 multilocular, 2 unilocular), 20 non syndromic OKCs (7 multilocular, 7 unilocular, 6 not informed), 21 ABs diagnosed according to the criteria proposed by the WHO (2017) [12]. Four dental follicles (DFs) served as controls. Only specimens with a mild inflammatory infiltrate or without an infiltrate were included. For $A B$, only the conventional clinical-radiographic type was selected. The study was approved by the Research Ethics Committee (Permit No. 1.091.779).

\section{Immunohistochemistry}

For immunohistochemistry, 3- $\mu$ m thick sections mounted on glass slides previously prepared with organosilane adhesive (Sigma, Munich, Germany) were deparaffinized and rehydrated. Endogenous peroxidase was blocked by incubating the sections with $3 \%$ hydrogen peroxide in phosphate-buffered saline (PBS) for $45 \mathrm{~min}$. The sections were incubated with citrate buffer, $\mathrm{pH} \mathrm{6.0,} \mathrm{for} 20 \mathrm{~min}$ in a water bath at $95^{\circ} \mathrm{C}$ for antigen retrieval and cooled for $20 \mathrm{~min}$ at room temperature. The sections were treated with $4 \%$ milk and incubated with Protein Block Serum-Free (Dako, Copenhagen, Denmark) for 10 min to block nonspecific reactions. Next, the sections were incubated with the primary antibodies diluted in antibody diluents with background reducing components (Dako) for 18 hours (overnight) at $4{ }^{\circ} \mathrm{C}$ (RANKL: N19, Santa Cruz, 1:150; OPG: N22, Santa Cruz, 1:150; CatK: 2F1, Abcam, 1:750; MMP8: MM0023-7, Santa Cruz, 1:50). The LSAB + System-HRP (Dako) was used for the detection of RANKL, OPG and CatK and the ADVANCE $^{\text {TM }}$ HRP system (Dako) for the detection of MMP8. The reactions were developed with $0.03 \%$ diamino benzidine (Dako) as chromogen and the slides were counterstained with Mayer's hematoxylin for 2 min. Central giant cell lesions were used as positive control for RANKL, OPG and CatK, and radicular cyst (RC) for MMP8. Sections incubated with serum diluent replacing the primary antibodies served as negative control.

The presence or absence of immunoreactivity in epithelium and connective tissue was analyzed, as well as the type of positive mesenchymal cell (fibroblast or endothelial cell). Immunoexpression in the 
epithelium was analyzed semi-quantitatively throughout the entire slide by two observers at $100 \mathrm{x}$ final magnification and scored as follows $[4,13,14]$ : 0 or no staining ( $<10 \%$ positive cells), 1 (11-25\% positive cells), 2 ( $26-50 \%$ positive cells), 3 ( $51-75 \%$ positive cells), and 4 ( $>75 \%$ positive cells). In connective tissue, the analysis was quantitative. The number of positive cells was counted in five representative histological fields corresponding to the hotspot areas for each marker at 400x final magnification, and the mean number was calculated for each case $[4,13,14]$. Areas containing inflammatory cells were avoided in the selection of the five histological fields. The image of each field was captured with a Nikon E200 photomicroscope. Immunostained cells were counted using the Cell Counter tool of the Image J for Windows software (version 3.0). In addition to cell counting, this tool marks positive and negative cells in different colors. The median number of positive cells was calculated for each lesion according to the protein analyzed.

\section{Statistical analysis}

Epithelial staining was compared by the $\mathrm{X}^{2}$ test and mesenchymal staining by the Kruskal-Wallis test. A level of significance of $5 \%$ was adopted $(p<0.05)$.

\section{Results}

Positive staining was generally observed in the cytoplasm of epithelial and connective tissue cells. The cell types that expressed the proteins studied in connective tissue were fibroblasts and endothelial cells.

Comparison of epithelial expression of the proteins studied among lesions (Table 1) revealed a significant difference for RANKL $(p<0.001)$. The highest immunostaining for this protein was found in OKC ( $75 \%$ of cases classified as score 4$)$ and $A B(61.9 \%$ of cases classified as score 4$)$. The expression of CatK also differed significantly $(p<0.001)$, with the highest expression in OKC ( $50 \%$ of cases classified as score 3 and $40 \%$ of cases classified as score 4 ), followed by GOC and AB. The highest expression of MMP8 was found in GOC ( $77.8 \%$ of cases classified as score 4$)$, followed by OKC $(p<0.001)$. Dental follicles exhibited the highest expression of OPG ( $100 \%$ of cases classified as score 4$)$, followed by DC $(88.9 \%$ as score 4$)(p=0.002)$. 
Table 1

Comparison of epithelial expression of the proteins studied between dentigerous cyst, glandular odontogenic cyst, odontogenic keratocyst, ameloblastoma, and dental follicle.

\begin{tabular}{|c|c|c|c|c|c|c|}
\hline Protein & Score & & & & & \\
\hline \multirow[t]{3}{*}{ RANKL } & 0 & 1 & 2 & 3 & 4 & $p$ \\
\hline & $(<10 \%)$ & (11 a $25 \%)$ & $(26$ a $50 \%)$ & (51 a 75\%) & $(>75 \%)$ & \\
\hline & n (\%) & $\mathrm{n}(\%)$ & n (\%) & $\mathrm{n}(\%)$ & $\mathrm{n}(\%)$ & \\
\hline $\mathrm{DC}$ & $0(0)$ & $1(11,1)$ & $3(33,3)$ & $3(33,3)$ & $2(22,2)$ & \\
\hline GOC & $0(0)$ & $1(11,1)$ & $3(33,3)$ & $3(33,3)$ & $2(22,2)$ & $<0,001$ \\
\hline OKC & $0(0)$ & $0(0)$ & $1(5)$ & $4(20)$ & $15(75)$ & \\
\hline$A B$ & $0(0)$ & $0(0)$ & $0(0)$ & $8(38,1)$ & $13(61,9)$ & \\
\hline DF & $3(75)$ & $1(25)$ & $0(0)$ & $0(0)$ & $0(0)$ & \\
\hline \multicolumn{7}{|l|}{ CatK } \\
\hline DC & $2(22,2)$ & $3(33,3)$ & $2(22,2)$ & $1(11,1)$ & $1(11,1)$ & \\
\hline GOC & $1(11,1)$ & $1(11,1)$ & $0(0)$ & $4(44,4)$ & $3(33,3)$ & $<0,001$ \\
\hline OKC & $0(0)$ & $0(0)$ & $2(10)$ & $10(50)$ & $8(40)$ & \\
\hline$A B$ & $6(28,6)$ & $1(4,8)$ & $8(38,1)$ & $2(9,5)$ & $4(19)$ & \\
\hline DF & $4(100)$ & $0(0)$ & $0(0)$ & $0(0)$ & $0(0)$ & \\
\hline \multicolumn{7}{|l|}{ MMP8 } \\
\hline DC & $0(0)$ & $1(11,1)$ & $1(11,1)$ & $5(55,6)$ & $2(22,2)$ & \\
\hline GOC & $0(0)$ & $0(0)$ & $0(0)$ & $2(22,2)$ & $7(77,8)$ & $<0,001$ \\
\hline OKC & $0(0)$ & $0(0)$ & $3(15)$ & $10(50)$ & $7(35)$ & \\
\hline$A B$ & $7(33,3)$ & $5(23,8)$ & $5(23,8)$ & $3(14,3)$ & $1(4,8)$ & \\
\hline DF & $4(100)$ & $0(0)$ & $0(0)$ & $0(0)$ & $0(0)$ & \\
\hline \multicolumn{7}{|l|}{ OPG } \\
\hline DC & $0(0)$ & $0(0)$ & $1(11,1)$ & $0(0)$ & $8(88,9)$ & \\
\hline GOC & $1(11,1)$ & $2(22,2)$ & $4(44,4)$ & $2(22,2)$ & $0(0)$ & 0,002 \\
\hline OKC & $0(0)$ & $0(0)$ & $2(10)$ & $5(25)$ & $13(65)$ & \\
\hline$A B$ & $0(0)$ & $8(38,1)$ & $8(38,1)$ & $5(25)$ & $0(0)$ & \\
\hline
\end{tabular}

DC: Dentigerous Cyst, GOC: Glandular Odontogenic Cyst, OKC: Odontogenic Keratocyst, AB Ameloblastoma, DF: Dental Follicle 


\begin{tabular}{|c|c|c|c|c|c|}
\hline Protein & Score & & & & \\
\hline DF & $0(0)$ & $0(0)$ & $0(0)$ & $0(0)$ & $4(100)$ \\
\hline
\end{tabular}

Comparison of immunohistochemical expression of the different proteins in connective tissue among lesions (Table 2) revealed higher expression of RANKL and CatK in OKC (median: 85.0 and 53.5, respectively) and $A B$ (median: 80.0 and 29.0) $(p<0.001)$. MMP8 was expressed at higher levels in OKC (median: 78.5), DC (median: 63.0) and GOC (median:46.0) $(\mathrm{p}<0.001)$. The highest expression of OPG was detected in OKC (median: 52.0), DF (median: 48.0), and DC (median: 27.0$)(p<0.001)$. 
Table 2

Comparison of immunohistochemical expression of the proteins studied in connective tissue between dentigerous cyst, glandular odontogenic cyst, odontogenic keratocyst, ameloblastoma, and dental follicle.

\begin{tabular}{|llll|}
\hline PROTEIN & Median & $\mathbf{Q}_{25-} \mathbf{Q}_{75}$ & $\boldsymbol{p}$ \\
\hline RANKL & & & $<0.001$ \\
DC & 40,0 & $20,5-58,5$ & \\
GOC & 25,0 & $1,5-48,5$ & \\
OKC & 85,0 & $40,25-92,0$ & \\
AB & 80,0 & $74,5-87,5$ & \\
DF & 0 & $0,0-3,0$ &
\end{tabular}

\section{CatK}

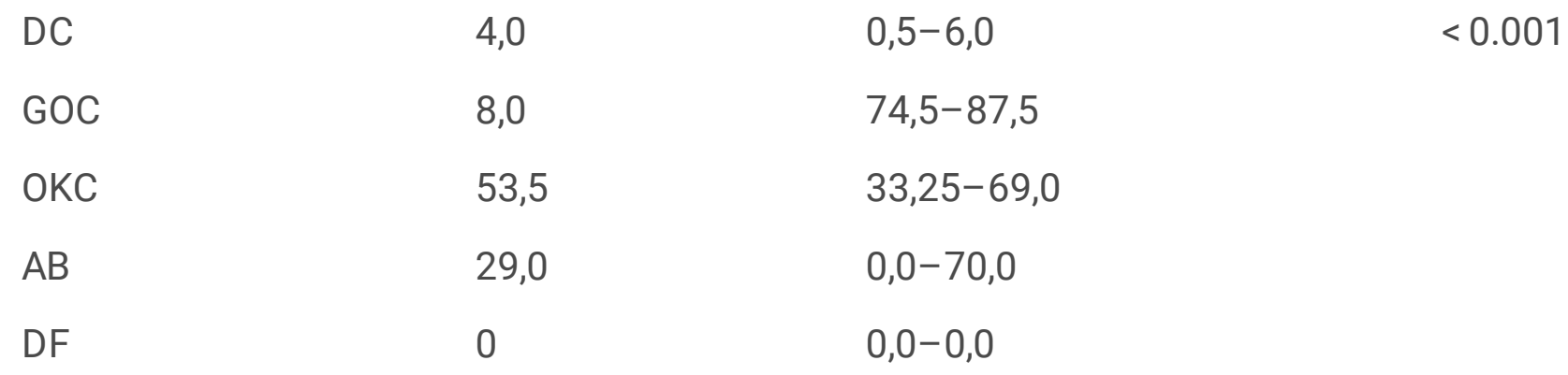

\section{MMP8}

DC

GOC

46,0

$31,0-64,0$

OKC

78,5

$66,75-84,25$

$A B$

17,0

$10,0-33,0$

DF

16,0

$10,75-43,0$

\section{OPG}

$\begin{array}{llll}\text { DC } & 27,0 & 17,0-59,5 & <0.001 \\ \text { GOC } & 3,0 & 0,0-13,0 \\ \text { OKC } & 52,0 & 33,5-57,75 \\ \text { AB } & 13,0 & 1,5-20,0 \\ \text { DF } & 48,0 & 39,75-58,5\end{array}$

DC: Dentigerous Cyst, GOC: Glandular Odontogenic Cyst, OKC: Odontogenic Keratocyst, AB: Ameloblastoma, DF: Dental Follicle 
Figures 1 and 2 illustrate the immunostaining for RANKL, CatK, MMP8 and OPG in the epithelial and mesenchymal components of DC, GOC, OKC, AB, and DF.

\section{Discussion}

Odontogenic lesions arise from the activation of epithelial (cysts and tumors) and/or ectomesenchymal (tumors) remnants of odontogenesis that persist in the bone. These remnants can be stimulated by proteins that activate biological events essential for the growth and progression of the lesion. One important event is osteoclastogenesis, which is directly linked to the establishment of intraosseous lesions. The molecular events involved in the bone resorption and growth of odontogenic lesions are not fully understood, but studies have shown that some proteins seem to influence the osteolytic activity and expansion of these lesions $[1,4,11,15]$. The present study suggests the contribution of RANKL, CatK, MMP8 and OPG during the development of DC, GOC, OKC, and AB.

Analysis of the immunoexpression of osteoclastogenic factors in DC demonstrated high expression of OPG. However, significant expression of MMP8 was also observed in this cyst. Marked expression of OPG in DC is expected because of the indolent nature of the lesion. OPG inhibits the proliferation and differentiation of osteoclasts and competes with RANKL by preventing its binding to RANK, thus inhibiting osteolytic activity $[7,15]$. Suojanen et al. (2016) found higher expression of OPG in DC when compared to $\mathrm{OKC}$ and $\mathrm{AB}$, in agreement with our results [16].

Studies investigating the expression of MMP8 in odontogenic lesions are scarce in the literature and there is only one study that involved DC [16]. Our findings are consistent with the results of Suojanen et al. (2016) who also found high immunoexpression of MMP8 in DC. MMP8 is one of the most important endopeptidases that mainly degrades collagen I $[16,17]$. By acting together with other factors that stimulate osteolytic activity, this protease would contribute to the growth and progression of intraosseous lesions. It is believe that the presence of MMP8 in CD evaluated in the present study was important for the lesion to begin. However the predominance of OPG is responsible for its indolent characteristic. With respect to CatK, low expression of this protein was detected in the DC cases. CatK participates in the degradation of bone matrix protein components, especially collagen I, the most abundant collagen in bone. However, the low expression of CatK in DC suggests a minor role of this protein in the development of this lesion. There are no studies evaluating the immunoexpression of CatK in DC.

In GOC, MMP8, RANKL, and CatK were expressed at higher levels than OPG. This finding may explain in part the aggressive nature of this cyst since it exhibits higher expression of molecules that favor bone resorption.

The highest immunoexpression of RANKL was observed in OKC. This result is consistent with the literature since OKC is considered a more aggressive lesion compared to other odontogenic cysts $[18,19]$ and is associated with higher bone resorption [20, 21]. Tekkesin, Mutlu and Oglac (2011), Matos et al. (2013) and Siar et al. (2015) also obtained high immunoexpression of RANKL in OKC $[1,11,15]$.

Furthermore, ours results show significant expression of OPG in the capsule of OKC. This result raises the 
possibility that, although considered an aggressive lesion, OKC normally possesses slower growth than a tumor lesion such as AB. Similar results have been reported by Tekkesin, Mutlu and Oglac (2011) who evaluated the levels of RANKL/OPG in OKC and found higher expression of OPG in $62.4 \%$ of the cases studied [11]. In contrast, in the capsule of OKC of our sample, OPG was less expressed than RANKL, CatK and MMP8. It is believed that the different proportions of stimulating and inhibiting factors favor greater or lower bone resorption.

Significant staining for CatK was observed in OKC, suggesting that this protein is important for the development of this lesion. The immunohistochemical expression of MMP8 in OKC was more significant in the capsule. There are no studies that evaluated the expression of CatK and MMP8 in OKC. However, some authors have demonstrated a relationship of CatK with higher bone resorption in the progression of rheumatoid arthritis and periodontitis [22], and of MMP8 with reduced bone repair and an increased risk of developing malignant tumors $[2,17]$.

Ameloblastoma parenchyma and stroma studied revealed high levels of RANKL, compatible with an aggressive lesion. Low expression of OPG was also observed in $A B$, which is consistent with the fact that this protein is an inhibitor of osteoclastogenesis. These results corroborate several studies in the literature $[1,11,23,24]$. Kumamoto and Ooya (2004) found higher expression of RANKL compared to OPG in the parenchyma of $A B$ [24]. Tekkesin, Mutlu and Oglac (2011) detected higher expression of RANKL in the stroma of $A B$ when compared to OPG [11].

In the present study, significant CatK staining was observed in the parenchyma and in the stroma of AB. Cathepsin $\mathrm{K}$ is a favorable biological marker of osteoclastogenesis which, together with RANKL, could explain the bone resorption and expansion potential of this lesion. Kim et al. (2014) observed important expression of CatK in peripheral ameloblastoma [25]. There are no previous studies evaluating the expression of CatK in intraosseous $A B$. The immunohistochemical expression of MMP8 in AB was not significant. The studies published so far did not investigate the participation of MMP8 in the pathogenesis of AB. However, other MMPs have been explored and an important role in the establishment, bone resorption and growth of this tumor has been demonstrated $[3,26]$.

Comparison of the immunohistochemical expression of the proteins between the lesions studied revealed an apparently greater participation of RANKL and CatK in the pathogenesis of OKC, $A B$ and GOC than in DC. This result might be important to explain the greater bone resorption, growth and aggressiveness associated with AB, OKC and GOC $[3,7,11]$. Tekkesin, Mutlu and Oglac (2011) evaluated the immunohistochemical expression of RANK, RANKL and OPG in OKC, AB and RC [2011]. In that study, the expression of RANKL was high and similar in $A B$ and OKC and low in RC. RANK expression was higher in OKC and OPG expression was low, without differences between lesions. Brito et al. (2018) evaluated the expression of the RANK/RANKL/OPG triad in unicystic ameloblastoma, OKC, and DC [27]. Higher expression of RANKL was observed in unicystic ameloblastoma, followed by OKC. The latter exhibited higher expression of OPG when compared to the other lesions studied. Higher expression of RANK and lower expression of RANKL were found in DC. The present study also compared the 
immunohistochemical expression of RANKL and OPG in DC, AB and OKC. Higher expression of RANKL was observed in $\mathrm{OKC}$, followed by $\mathrm{AB}$. Taken together, these results suggest considerable osteolytic activity in $A B$ and $O K C$, as indicated by the high expression of factors that stimulate osteoclastogenesis.

Higher immunoexpression of OPG was observed in DC and DF. This finding might explain the lower osteolytic activity associated with these processes. Likewise, Tekkesin, Mutlu and Oglac (2011) found high immunopositivity for OPG in $70 \%$ of DF cases when compared to $A B, O K C$ and $D C$ [11]. According to Moraes et al. (2011) and Siar et al. (2015), higher expression of OPG is expected in more indolent lesions because of the inhibitory activity of this protein on osteoclastogenesis $[4,15]$.

Our results indicate higher expression of RANKL and CatK in lesions with a more aggressive behavior such as OKC, AB and GOC. OPG was more expressed in DF and DC and seems to be one of the molecules responsible for the slower growth of DC. MMP8 appears to play an important role in the growth of the GOC and OKC. Further studies involving odontogenic lesions and these markers, especially CatK and MMP8, are necessary to increase our understanding of the process of bone resorption in these lesions.

\section{Abbreviations}

DC: dentigerous cyst; GOC: glandular odontogenic cyst; OKC: odontogenic keratocyst; $A B$ :

ameloblastoma; RANK: receptor activator of nuclear factor kappa- $\beta$; RANKL: Receptor activator of nuclear factor kappa-B ligand; CatK: cathepsin K; MMP8: Matrix Metallopeptidase 8; OPG: osteoprotegerin; DF: dental follicles; PBS: phosphate-buffered saline;

\section{Declarations}

\section{ETHICS APPROVAL}

The study was approved by the Research Ethics Committee of Federal University of Bahia (Permit No. 1.091.779).

\section{AVAILABILITY OF DATA AND MATERIALS}

The datasets used and/or analysed during the current study are available from the corresponding author on reasonable request. All data generated or analysed during this study are included in this published article [and its supplementary information files.

\section{COMPETING INTERESTS}

The authors declare they have no competing interests

\section{ACKNOWLEDGEMENTS}


The authors thank Conselho Nacional de Desenvolvimento Científico e Tecnológico - Brasil (CNPQ) and Coordenação de Aperfeiçoamento de Pessoal de Nível Superior - Brasil (CAPES) for financial support. The authors declare that they have no financial affiliation or involvement with any commercial organization. The authors deny any conflicts of interest related to this study.

\section{AUTHORS' CONTRIBUTION}

VR and AC were major contributor in writing the manuscript. EG and MV carried out the laboratory development of this research. JF and RA were contributor in development of this research. AL carried out the analysis of the results and statistics. AC, JN and FC performed the histological examination. All authors read and approved the final manuscript.

\section{References}

1. Matos FR, De Moraes M, Das Neves SEB, et al. Immunohistochemical detection of receptor activator nuclear kb ligand and osteoprotegerin iç;’n odontogenic cysts and tumors. Oral Maxillofac. 2013; 71(11): 1886-1892.

2. Omar AAH, Haglund C, Virolainen S, Hayry V, et al. MMP-7, MMP-8 and MMP-9 in oral and cutaneous squamous cell carcinomas. J. Oral Maxillofac. Pathol. 2015; 19 (4): 459-468.

3. Dutra K L, Cordeiro MMR, Vieira DSC, et al. Immunohistochemical expression of matrix metalloproteinases in ameloblastomas and pericoronal follicles. Oral Pathol. Med. 2016; 45(8): 586590.

4. Moraes $M$, Matos FR, Souza LB, et al. Immunoexpression of RANK, RANKL, OPG, VEGF, and vWF in radicular and dentigerous cysts. Oral Pathol. Med. 2011; 42: 468-473.

5. ChenB, Wu W, Sun W, et al. RANKL expression in periodontal disease: Where does RANKL come from? BioMed research international. 2014; 4:150-159.

6. Guerrini MM, Takayanagi H. The immune system, bone and RANKL. Arch. Biochem. 2014; 561: 118123.

7. Sun $\mathrm{H}, \mathrm{Li} \mathrm{Q}$, Zhang $\mathrm{Y}$, et al. Regulation of OPG and RANKL expressed by human dental follicle cells in osteoclastogenesis. Cell Tissue Res. 2015; 362(2): 399-405.

8. Hua Y, Robinson TJ, Cao Y, et al. Cathepsin $\mathrm{K}$ knockout alleviates aging-induced cardiac dysfunction. Aging Cell. 2015; 14(3): 345-351.

9. Guha M, Srinivasan S, Koenigstein A, et al. Enhanced osteoclastogenesis by mitochondrial retrograde signaling through transcriptional activation of the cathepsin K gene. N. Y. Acad. Sci. 2016; 1364:5261.

10. Gao B, Chen W, Hao L, et al. Inhibiting periapical lesions through AAV-RNAi silencing of cathepsin K. Dent. 2013; 92(2): 180 -186.

11. Tekkesin MS, Mutlu S, Olgac V. The Role of RANK/RANKL/OPG Signalling pathways in osteoclastogenesis in odontogenic keratocysts, radicular cysts, and ameloblastomas. Head Neck 
Pathol. 2011; 5(3): 248-253.

12. El-Naggar AK, Chan JKC, Grandis JR, et al. World Health Organization Classification of Tumours. Pathology and Genetics of Head and Neck Tumours. 2017; $4^{\text {th }}$ Lyon: IARC Press.

13. Sá MC, Matos FR, Conceição TS, et al. Immunoexpression of tumour necrosis factor-a, interleukin 1$a$ and interleukin-10 on odontogenic cysts and tumours. Int. Endod. J. 2016; 10(3): 45-51.

14. Santos SCLT, Couto LA, Fonseca JM, et al. Participation of osteoclastogenic factors in immunopathogenesis of human chronic periapical lesions. Oral Pathol. Med. 2017; 3:1-7.

15. Siar $\mathrm{CH}$, Tsujigiwa $\mathrm{H}$, Ishak I, et al. RANK, RANKL, and OPG in recurrent solid/multicystic ameloblastoma: Their distribution patterns and biologic significance. Oral Surg. Oral Med. Oral Pathol. Oral Radiol. 2015; 119(1): 83-91.

16. Suojanen H, Letonem N, Farkkila E, et al. Common matrix metalloproteinases (MMP8, $-9,-25$ and -26 ) cannot explain dentigerous cyst expansion. J. Clin. Diagnostic Res. 2014; 8(9): 82-85.

17. Lawal A, Adisa A, Kolude B, et al. Immunohistochemical expression of MMP-2 and MMP-8 in oral squamous cell carcinoma. Clin. Exp. Dent. 2015; 7(2): e203-e207.

18. Pogrel MA. The keratocystic odontogenic tumour (KCOT) - An odyssey. J. Oral Maxillofac. Surg. 2015; 44(12): 1565-1568.

19. Leung YY, Lau SL, Tsoi KY, et al. Results of the treatment of keratocystic odontogenic tumours using enucleation and treatment of the residual bony defect with Carnoy's solution. J. Oral Maxillofac. Surg. 2016; 45(9): 1154-1158.

20. Barnes L, Eveson JW, Reichart P, et al. World Health Organization Classification of Tumours. Pathology and Genetics of Head and Neck Tumours. 2005; Lyon: IARC Press.

21. Stoelinga PJW. The management of aggressive cysts of the jaws. Oral Maxillof. 2012; 11(1): 2-12.

22. Hao L, Zhu G, Lu Y, et al. Deficiency of cathepsin K prevents inflammation and bone erosion in rheumatoid arthritis and periodontitis and reveals its shared osteoimmune role. FEBS Letters. 2015; 589(12): $1331-1339$.

23. Bansal S, Desai RS, Shirsat P, et al. The occurrence and pattern of ameloblastoma in children and adolescents: An Indian institutional study of 41 years and review of the literature. J. Oral Maxillofac. Surg. 2015; 44(6): 725-731.

24. Kumamoto H, Ooya K. Expression of parathyroid hormone-related protein (PTHrP) osteoclast differentiation factor (ODF)/receptor activator of nuclear factor kappa B ligand (RANKL) and osteoclastogenesis inhibitory factor (OCIF)/ osteoprotegerin (OPG) in ameloblastomas. Oral Pathol. Med. 2004; 33: 46-52.

25. Kim YS, Lee SK. Different protein expressions between peripheral ameloblastoma and oral basal cell carcinoma occurred at the same mandibular molar area. Korean J 2014; 48: 151- 158.

26. Henriques ÁCG, Vasconcelos MG, Galvão HC, et al. Comparative analysis of the immunohistochemical expression of collagen IV, MMP-9, and TIMP-2 in odontogenic cysts and tumors Oral Surg. Oral Med. Oral Pathol. Oral Radiol Endod. 2011; 112(4): 468-475. 
27. Brito-Mendoza L, Bologna-Molina R, Irigoyen-Camacho ME, et al. A comparison of Ki67, sSyndecan-1 (CD138), and molecular RANK, RANKL, and OPG triad expression in odontogenic keratocytes, unicystic ameloblastoma, and dentigerous cysts. Dis Markers. 2018; 29: 1-7.

\section{Figures}

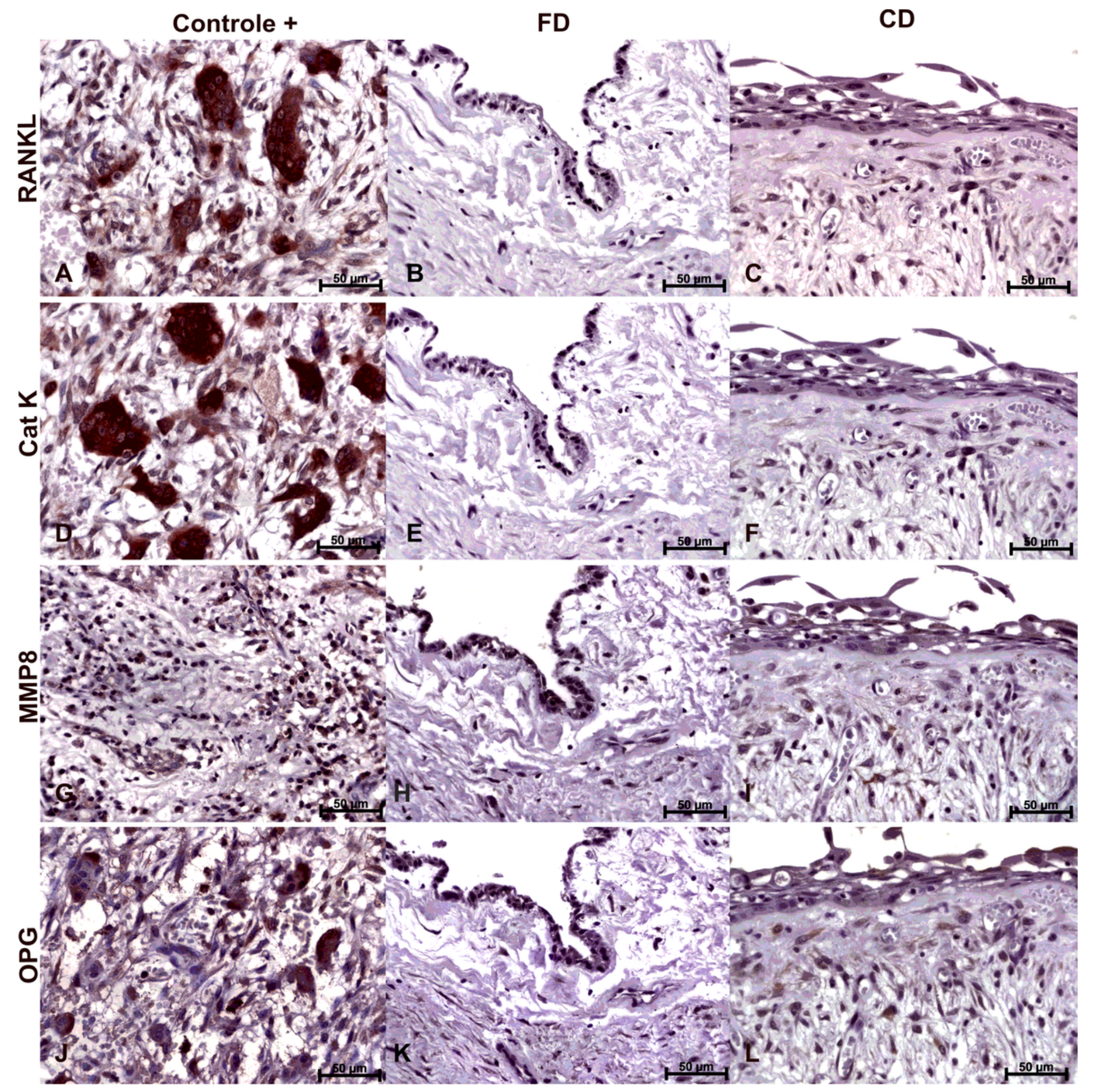

Figure 1 
Immunoexpression of RANKL, CatK, MMP8 and OPG in dental follicle (DF), dentigerous cyst (DC), and positive control (A, D and J: central giant cell lesion, G: radicular cyst). Note the absence of expression of RANKL (B) and CatK (E) in DF and weak expression in the cystic epithelium and capsule of DC (C and F). Expression of OPG is more evident in the cytoplasm and nucleus of epithelial and mesenchymal cells of DF (K) and DC (L). MMP8 present in the cytoplasm and nucleus of mesenchymal cells of DF (I).

\section{COG}
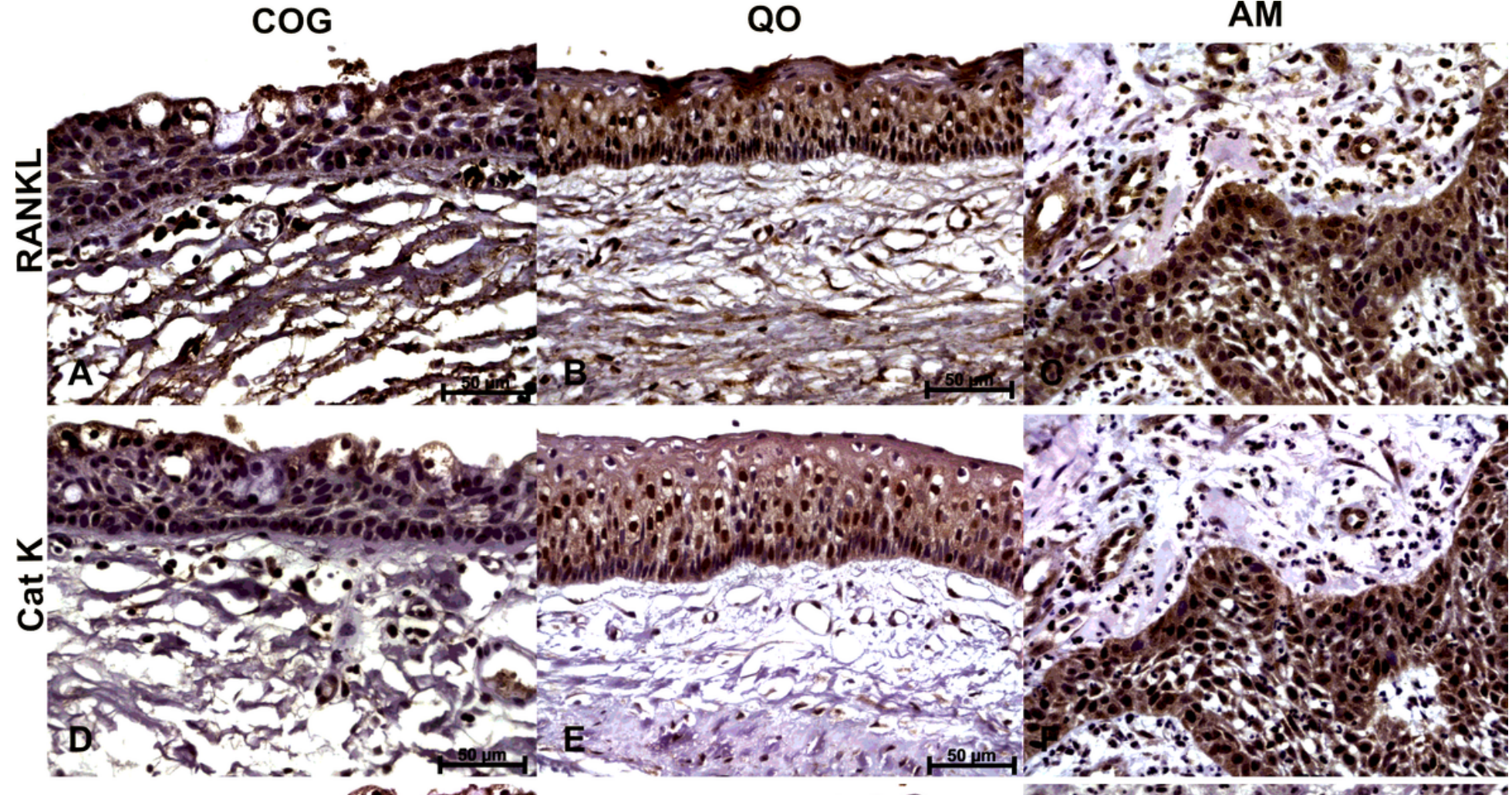

QO

AM

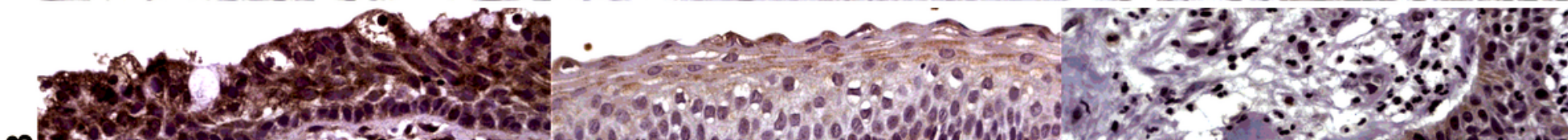

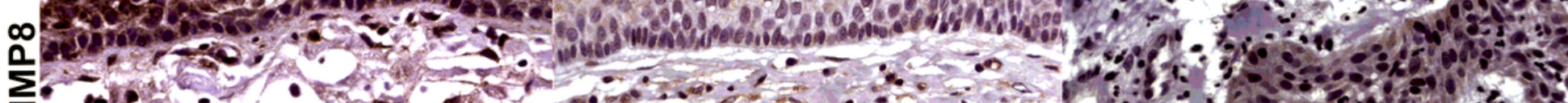

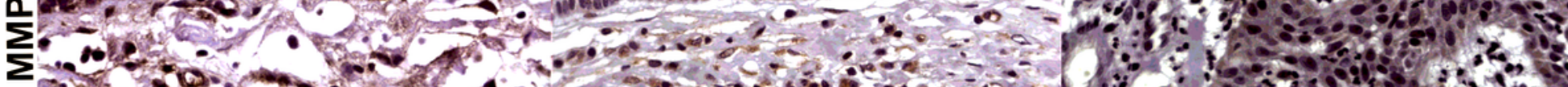

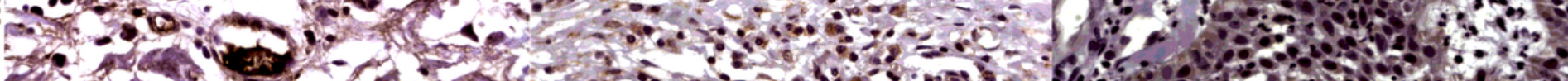

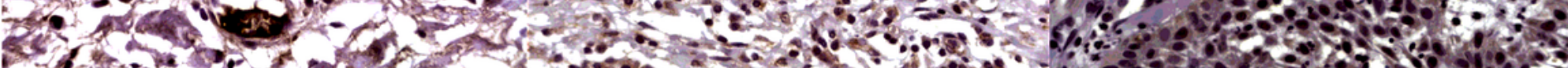

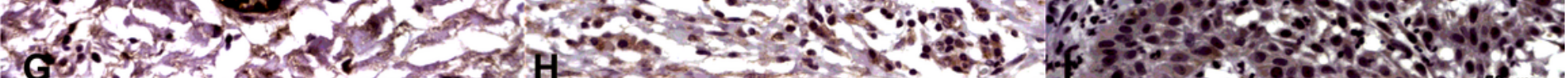

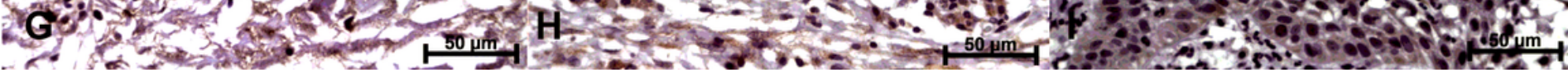

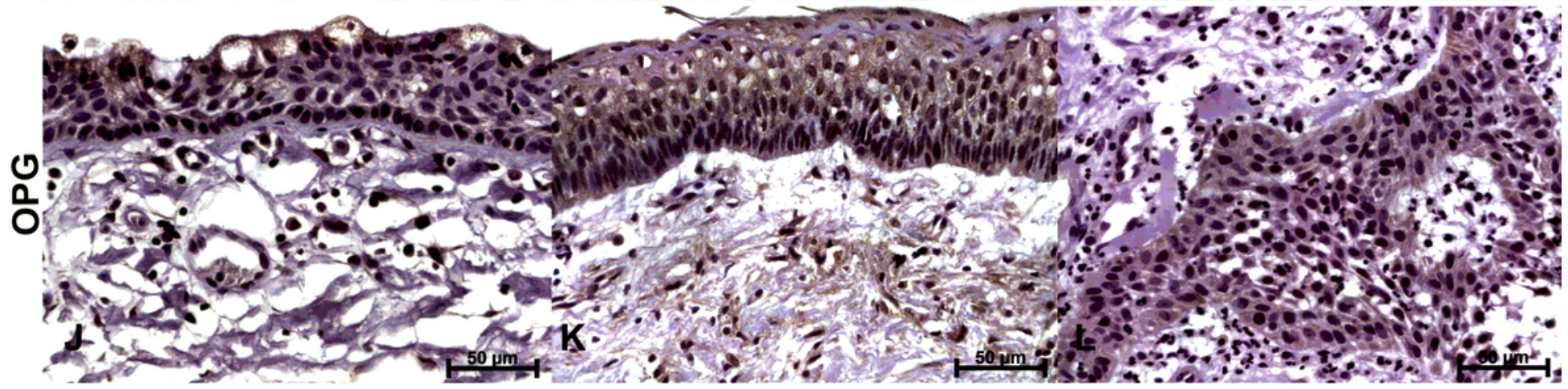

Figure 2 
Immunoexpression of RANKL, CatK, MMP8 and OPG in glandular odontogenic cyst (GOC), odontogenic keratocyst (OKC), and ameloblastoma (AB). Note the high expression of RANKL (C) and CatK (F) in the parenchyma and stroma of $A B$. High expression levels are seen in the cystic epithelium and capsule of OKC (B and $E$ ) and lower expression levels in GOC ( $A$ and $D)$. Expression of MMP8 is evident in the cytoplasm and nucleus of epithelial and mesenchymal cells of GOC (G, OKC $(H)$ and $A B(I)$. OPG is less evident in $\mathrm{GOC}(\mathrm{J}), \mathrm{OKC}(\mathrm{K})$ and $A B(\mathrm{~L})$. 